\title{
Contraction of westward-travelling nonlocal modons due to the vorticity filament emission
}

\author{
D. Berson ${ }^{1}$ and Z. Kizner ${ }^{1,2}$ \\ ${ }^{1}$ Department of Physics, Bar-Ilan University, Ramat-Gan 52900, Israel \\ ${ }^{2}$ Department of Mathematics, Bar-Ilan University, Ramat-Gan 52900, Israel
}

Received: 5 September 2001 - Accepted: 30 September 2001

\begin{abstract}
Long-term evolution of westward-travelling nonlocal modons on the $\beta$-plane, i.e. dipolar vortices imbedded in slowly damping Rossby wave fields, is studied numerically. In the framework of the nondivergent (barotropic) model, two stages of the evolution are observed. At the first stage (for about 30 synoptic periods), the parameters and the form of the vortex practically remain constant, whereas at the second stage, vorticity filaments are emitted. Due to the filamentation, the vortex core contracts, the potential vorticity peaks of the vortex pair get closer, and the modon speeds up. In the divergent (equivalent-barotropic) model, nonlocal modons and the Lamb modon (that has no wave field outside the dipolar core) evolve much more slowly, essentially preserving the initial shape and propagation speed until about 100 synoptic periods.
\end{abstract}

\section{Introduction}

Vortex pairs consisting of two oppositely signed regions of vorticity that travel as a single entity represent one of the main types of coherent structures in geophysical fluid dynamics. The existence of a significant dipolar component in the observed synoptic eddies in the ocean (the so-called mushroom structures) was clearly confirmed by remote sensing imaginary (Ginsburg and Fedorov, 1984; Ikeda et al., 1984; Ikeda and Emery, 1985; Ahnlas et al., 1987; Fedorov and Ginsburg, 1989; Johannessen et al., 1989; Hooker et al., 1995). In laboratory experiments, vortical dipoles were observed in many circumstances. For example, paired vortices may appear as a well-organized product of a pulsed turbulent jet (van Heijst and Flór, 1989; Flierl, et al., 1983; Flór and van Heijst, 1994), in the wake of a cylinder moving through a soap film (Couder and Basdevant, 1986) or through a rotating fluid (Velasco Fuentes and van Heijst, 1994). Electric pulses in a layer of mercury, subjected to a magnetic field to make the motions two-dimensional, can also lead to the

Correspondence to: Z. Kizner (zinovyk@mail.biu.ac.il) formation of dipolar vortices (Nguyen Duc and Sommeria, 1988). High-resolution numerical simulations also show the emergence of dipolar vortices from unstable structures both on the $f$-plane (Orlandy et al., 1994) and $\beta$-plane (Kizner and Berson, 2000, hereafter KB).

In the framework of a nondivergent (rigid-lid condition), barotropic model on the $\beta$ - plane, Stern (1975) derived an exact standing localized dipolar solution and suggested the term "modon" to designate it. The vorticity in Stern's modon is confined within a certain circular domain, equals zero outside this region and jumps at its boundary. Larichev and Reznik (1976) have considered a divergent (equivalent-barotropic), quasi-geostrophic model and found an exact localized dipolar solution that can be understood as a generalization of Stern's modons for the case of propagating vortices with a continuous vorticity field. Below, we will refer to solutions of such a degree of smoothness as high-smoothness ones, while the abbreviation LR will be used to refer to the publication of Larichev and Reznik (1976) and their specific modon solution. In fact, the first high-smoothness modon, which served as a prototype for Stern's and LR constructions, was that suggested by Lamb (1932), who provided an example of a steadily propagating dipolar solution to the classical Euler equations of the two-dimensional inviscid fluid dynamics. The cited works of Stern and LR had a significant impact and initiated a series of subsequent publications treating barotropic, two-layer, or three-dimensional modons (e.g. Flierl et al., 1980; Berestov, 1979, 1981; Kizner, 1984, 1986a, b, 1988, 1997; Reznik, 1985; Reznik and Sutyrin, 2001; Pakyari and Nycander, 1996).

Verkley and Tribbia have extended the modon concept to spherical geometry. Superimposing the dipole and monopole stream functions, Verkley $(1984 ; 1987)$ constructed a nondivergent, high-smoothness westward-propagating modon on the sphere, while Tribia (1984) found a similar divergent modon. Boyd (1994) adopted spherical solutions of Verkley and Tribbia to obtain the so-called nonlocal modons for the case of the $\beta$-plane. In contrast with the localized modons of Lamb (1932), Stern (1975), LR, and Flierl et al. (1980) 
that decay more or less rapidly outside the matching circle, thus providing finite integral characteristics (such as the total vorticity, energy and entrophy), when the nonlocal modons decay too slowly in the above sense. Nonlocal modons are actually vortical cores of essentially nonlinear Rossby waves. In the travelling frame attached to such a modon, the latter is characterized by two different proportionalities between the potential vorticity (PV) and the stream function, inside some circle, where the main vorticity is concentrated, and outside it, in the wave field area.

$\mathrm{KB}$ showed numerically that, on the $\beta$-plane under the rigid-lid condition (nondivergent case), multipolar vortices given by the solutions of Stern and LR, the so-called shielded modons, as well as some non-stationary vortical structures, collapse, giving birth to the eastward-propagating LR dipoles and the westward-propagating vortical structures. The latter demonstrated remarkable persistence (surviving head-on collisions with LR modons), and were identified as nonlocal modons. It should be noted that, in addition to the generaltype nonlocal modons, Boyd (1994) also considered a special case of "minimum amplitude" solutions, in which he imposed a restriction on the parameters by minimizing the external wave field. A reason for such a consideration is stated in his later publications (Boyd, 1998a, b): in some nonlinear wave equations important in fluid dynamics, particle physics and nonlinear optics (such as the fifth-order Korteweg-deVries equation, the so-called $\phi^{4}$ model and the third-order Schrödinger equation), when a nonlocal structure evolves from a localized pulse the far field at large times must be of minimum amplitude. However, no similar (or any other definite) constraints upon the modon parameters were revealed using the identification procedure of $\mathrm{KB}$ (true, in the experiments of $\mathrm{KB}$, the evolution of the vortex was followed for a few to tens of synoptic periods, whereas according to Boyd (1998b), the far field takes a long time to settle into the theoretically predicted steady state). The asymptotic solution of Flierl and Haines (1994) constructed under the rigid-lid condition and representing a strongly nonlinear westwardpropagating vortical pair surrounded with a weak wave field has much in common with the nonlocal modon of Boyd, but strictly speaking, the vorticity is discontinuous in this solution.

In contrast to the localized modons travelling either westward at supercritical speeds (faster than the Rossby waves) or eastward, the nonlocal modons on the $\beta$-plane propagate westward at subcritical speeds (slower than the longest Rossby waves). As will be shown in the present paper, the solution that discriminates between LR and the nonlocal modons, i.e. the vortex pair that travels westwards exactly at the critical speed, is the Lamb modon adapted to the $\beta$-plane.

Persistence of the eastward-propagating modons was shown using numerical techniques by McWilliams et al. (1981), McWilliams and Zabusky (1982), and Larichev and Reznik (1982, 1983). Numerical simulations of Makino et al. (1981), Zabusky and McWilliams (1982), and Hesthaven et al. (1993) revealed the important difference between the eastward- and the westward-travelling localized modons: the eastward-travelling LR modons were shown to be stable (at least within the limits, in which stability can be studied numerically), whereas the westward-travelling localized modons turned out to be unstable to the tilt perturbations, i.e. when launched with some angle to the east-west axis. Nycander (1992) explained the mechanism of the tilt instability of the westward-propagating vortex through the $\beta$-effect, while Velasco Fuentes and Van Hejst (1994) have proved the difference between the eastward- and the westward-travelling dipoles in a series of laboratory experiments. Their results were similar to those of Hesthaven et al. (1993) and clearly showed that a small deviation of an eastward-travelling modon from its stationary pathway results in small oscillations of the modon's trajectory around the equillibrium "latitude", whereas, in the case of a westward-travelling modon, such a perturbation implies large displacements of the dipole trajectory in the northern or southern directions. Despite that in the cited papers of Nycander (1992) and Hesthaven et al. (1993) the problem of tilt instability was considered in relation to the divergent localized westward-travelling modons, their main results remain valid when considering nonlocal modons (KB).

Many researchers explain "blocking" events in the atmosphere by westward-travelling dipolar vortices (e.g. McWilliams, 1980; Tribia, 1984; Haines and Marshall, 1987; Haines, 1989; Butchart et al., 1989; Verkley, 1984, 1987, 1990, 1993; Haupt et al., 1993). Others argue that, due to their instability, the westward-propagating dipole vortices are unlikely to be the cause of blocking (Nycander, 1992; Hesthaven et al., 1993; DiBatista and Polvani, 1998). In the case of nonlocal modons, in addition to the tilt instability, the Rossby wave radiation must be taken into consideration. All of these arguements make a thorough study of the evolution of the nonlocal modons especially important.

Our previous experiments (KB) lead to the conclusion that, when moderately tilted, nonlocal modons may drift mainly westwards for a few to tens of synoptic periods - a time sufficient for a blocking phenomenon to appear. There is, however, another more delicate problem. As shown by KB, even if only anti-symmetric perturbations are permitted, i.e. when the nonlocal modon propagates exactly westward, it emits vorticity filaments. When this filamentation becomes sufficiently strong (or lasts long enough), it must affect the vortex evolution process. Therefore, the main question is: what is the effect of the vorticity filamentation on the form, translation speed and other parameters of the nonlocal modons?

The first study devoted to the evolution of the westwardtravelling vortex pairs on the $\beta$-plane under the rigid-lid condition was that of Flierl and Haines (1994). They initialized their numerical model by using the westward-propagating Lamb modon (which has no wave periphery outside the vortical core). Due to Rossby wave radiation, a wave periphery developed rapidly and the vortex evolved into a quasishape-preserving nonlocal modon. The computations, however, were performed for the weak $\beta$-effect and covered the initial stage of the evolution, when the filamentation was undetectable. 
In the present work, we focus on the long-term evolution of the nonlocal modons in the presence of a $\beta$-effect, which is not regarded as small. In the framework of the nondivergent model, two main stages of the evolution of a moderately nonlinear, nonlocal modon are observed. The first, quasishape-preserving stage is similar to the dynamics described by Flierl and Haines (1994) and lasts for about 30 synoptic periods. During the second stage (a few tens of synoptic periods at least), due to the filamentation, the vortex core contracts considerably (i.e. its radius decreases) and speeds up, but the vortex remains essentially of the nonlocal modon type.

In the framework of the equivalent-barotropic model, nonlocal modons prove to be even more persistent: their shapepreserving propagation lasts for about 100 synoptic periods, the subsequent filamentation being relatively weak. However, the question remains as to whether a speeding-up divergent, nonlocal modon (if its initial translation speed is sufficiently high) may evolve into the Lamb modon. Similarly, if the Lamb modon emits filaments, will it transform into a LR modon? These questions are also addressed in the present paper and none of the above scenarios is found to be realistic. An interesting result is that the closer the nonlocal modon is to the Lamb modon, the more robust it is. On the $f$-plane, the Lamb modon is known for its high robustness (van Geffen and van Heijst 1998; Babkin et al., 2000). This is why it is not surprising that, in our $\beta$-plane experiments, it turned out to be very persistent (tilt instability apart).

\section{Formulation}

The nondivergent barotropic quasi-geostrophic equation and the equivalent-barotropic equation (in which the divergence of the horizontal currents appears) can be regarded as two opposite limiting cases of the two-layer quasi-geostrophic model. The first corresponds to the case of zero depth of the lower layer, while the second describes the currents in the upper layer under the assumption that the lower layer is infinitely deep (i.e. when the movements in the lower layer can be neglected). In terms of stream function $\psi$, the quasigeostrophic PV $q$ can be represented as

$q=\nabla^{2} \psi-m^{2} \psi+\beta y$,

where $m^{2}=0$ and $m^{2}=f_{0}^{2} /\left(g^{\prime} h\right) \neq 0$ correspond to the first (nondivergent) and the second (divergent) cases, respectively, while the conservation of $q$ is described by the equation

$$
\frac{\partial q}{\partial t}+\frac{\partial(\psi, q)}{\partial(x, y)}=0
$$

where $t$ is time; $x$ and $y$ are the zonal and meridional coordinates, respectively; $\nabla^{2}$ is the Laplacian in the horizontal plane; $\partial(,) / \partial(x, y)$ is the Jacobian of the corresponding functions with respect to $x$ and $y ; f=f_{0}+\beta y$ is the Coriolis parameter, $f_{0}$ and $\beta$ are constants (the $\beta$-plane model); $h$ is the unperturbed depth of the upper layer; and $g^{\prime}$ is the reduced gravity.
Let us consider a form-preserving current system travelling in the zonal direction at constant translation speed $U$. For such structures, in the travelling reference frame $\xi=x-U t, y$, Eq. (2) can be simplified to

$$
\frac{\partial}{\partial(\xi, y)}(\psi+U y, q)=0
$$

where the differentiations are carried out with respect to $\xi$ instead of $x$ (LR; Kamenkovich et al., 1986). The physical meaning of Eq. (3) is that the quasi-geostrophic vorticity is constant along the contours of the full stream function (taken in the moving reference frame), $\Psi=\psi+U y$. When relatively compact structures are considered, characterized by damping of $\psi$ at $\xi \rightarrow \infty$, the specific dependence of $q$ upon $\Psi$ outside some closed contour $q=$ const. can be shown to be simple proportionality $q= \pm l^{2} \Psi$, so that $U= \pm \beta / l$ (LR). Following Lamb (1932), Stern (1975) and LR, we will assume this contour to be a circle, inside which the dependence of $q$ on $\Psi$ is also proportionality:

$$
\Delta \psi-m^{2} \psi+\beta y= \begin{cases}-k^{2}(\psi+U y), & r<r_{0} \\ \pm l^{2}(\psi+U y), & r>r_{0} .\end{cases}
$$

Here, $r=\sqrt{\xi^{2}+y^{2}}$; parameters $k, l$ and $r_{0}$ are constants. Solutions to Eq. (4) can be shown to be localized (i.e. damping fast enough at $r \rightarrow \infty$ ) if $l^{2}$ in Eq. (4) bears a plus sign, in which case $U>0$ or, alternatively, if $l^{2}$ bears a minus sign and $U<-\beta / m^{2}$ (LR). The first solution of Eq. (4) for the case $m^{2}=0$ and $U=0$ was suggested by Stern (1975). This is not a high-smoothness solution, since its exterior and interior vorticity fields do not match at the contour $r=r_{0}$. The only high-smoothness localized solution of Eq. (4) known to date is the LR modon, which, in the polar coordinate system, $r, \theta$, can be presented in the form:

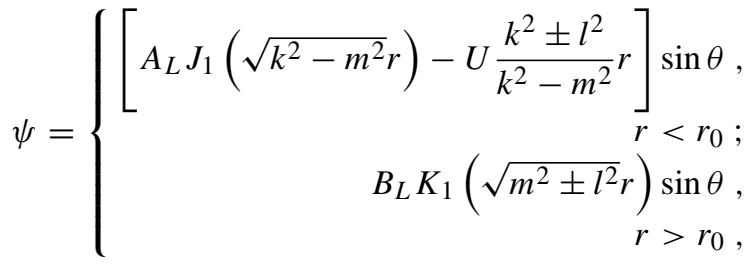

where $U= \pm \beta / l^{2}$ and $A_{L}, B_{L}$ are constants (if $l^{2}$ bears a minus sign $m^{2}>l^{2}$ ). Hereinafter, $J_{v}, K_{v}$ and $N_{v}$ are the $\nu$-order Bessel, McDonald and Neuman functions. The conditions of matching $\psi, \partial \psi / \partial r$ and $\partial^{2} \psi / \partial r^{2}$ at $r=r_{0}$ determine the coefficients $A_{L}$ and $B_{L}$ (as functions of $r_{0}, l$ and $k$ ) and impose the so-called "dispersion relationship" on the 
parameters $r_{0}, l, k$ and $m$ :

$$
\begin{aligned}
A_{L} & =-U \frac{\left(m^{2} \pm l^{2}\right) r_{0}}{\left(k^{2}-m^{2}\right) J_{1}\left(\sqrt{k^{2}-m^{2}} r_{0}\right)}, \\
B_{L} & =-U \frac{r_{0}}{K_{1}\left(\sqrt{m^{2} \pm l^{2}} r_{0}\right)}, \\
& -\frac{J_{2}\left(\sqrt{k^{2}-m^{2}} r_{0}\right)}{\sqrt{k^{2}-m^{2}} J_{1}\left(\sqrt{k^{2}-m^{2}} r_{0}\right)} \\
& =\frac{K_{2}\left(\sqrt{m^{2} \pm l^{2}} r_{0}\right)}{\sqrt{m^{2} \pm l^{2}} K_{1}\left(\sqrt{m^{2} \pm l^{2}} r_{0}\right)} .
\end{aligned}
$$

The characteristic property of the LR modon is that at $r \rightarrow$ $\infty$, this solution drops off exponentially.

As mentioned in the Introduction, stability of the eastward-travelling dipolar (non-shielded) LR modons can be regarded as proven numerically (McWilliams et al., 1981; Larichev and Reznik, 1982, 1983; McWilliams and Zabusky, 1982). In contrast, the shielded LR modons were shown to be unstable (KB): they radiate high-smoothness vortical dipoles travelling both east (LR dipoles) and west (nonlocal dipoles). The latter obey Eq. (4), where $l^{2}$ bears a minus sign. In the general case, in which $m^{2}$ may be non-zero, but $m^{2}<l^{2}$, the high-smoothness solutions of this category are given by the following formula:

$$
\psi=\left\{\begin{array}{c}
{\left[A_{N} J_{1}\left(\sqrt{k^{2}-m^{2}} r\right)-U \frac{k^{2}+l^{2}}{k^{2}-m^{2}} r\right] \sin \theta,} \\
r<r_{0} \\
{\left[B_{N} J_{1}\left(\sqrt{l^{2}-m^{2}} r\right)\right.} \\
\left.+C_{N} N_{1}\left(\sqrt{l^{2}-m^{2}} r\right)\right] \sin \theta \\
r>r_{0},
\end{array}\right.
$$

where again, $U=-\beta / l^{2}$ and $A_{N}, B_{N}, C_{N}$ are constants (Boyd, 1994). A similar strongly nonlinear solution was studied by Flierl and Haines (1994) for the special case of $m^{2}=0, B_{N}=0$. The solution they studied was, strictly speaking, a low-smoothness one, as they used the dispersion relationshipship of Lamb (1932), $J_{1}\left(k r_{0}\right)=0$, for a dipole on the $f$-plane (an asymptotic approach was applied).

Again, the conditions of matching $\psi, \partial \psi / \partial r$ and $\partial^{2} \psi / \partial r^{2}$ at $r=r_{0}$ determine the coefficients $A_{N}, B_{N}$ and $C_{N}$ as functions of $r_{0}, l$ and $k$ :

$$
\begin{aligned}
A_{N} & =\frac{\beta r_{0} \lambda^{2}}{l^{2} \kappa^{2} J_{1}\left(\kappa r_{0}\right)}=-U \frac{r_{0} \lambda^{2}}{\kappa^{2} J_{1}\left(\kappa r_{0}\right)}, \\
B_{N} & =\frac{A_{N}}{D}\left[\lambda J_{2}\left(\kappa r_{0}\right) N_{1}\left(\lambda r_{0}\right)-\kappa J_{1}\left(\kappa r_{0}\right) N_{2}\left(\lambda r_{0}\right)\right], \\
C_{N} & =\frac{A_{N}}{D}\left[\kappa J_{2}\left(\lambda r_{0}\right) J_{1}\left(\kappa r_{0}\right)-\lambda J_{1}\left(\lambda r_{0}\right) J_{2}\left(\kappa r_{0}\right)\right],
\end{aligned}
$$

where

$D=\frac{\lambda^{2}}{\kappa}\left[J_{2}\left(\lambda r_{0}\right) N_{1}\left(\lambda r_{0}\right)-J_{1}\left(\lambda r_{0}\right) N_{2}\left(\lambda r_{0}\right)\right]$, $\kappa=\sqrt{k^{2}-m^{2}}$ and $\lambda=\sqrt{l^{2}-m^{2}}$; subscript ' $N$ ' designates that here we are dealing with a nonlocal modon. Note that the only constraints imposed upon the parameters $r_{0}, l$ and $k$ of the solution (6) are that $m^{2}<k^{2}, m^{2}<l^{2}$ and $J_{1}\left(\kappa r_{0}\right) \neq 0$ (contrary to the condition applied by Flierl and Haines (1994) and the minimization of the external wave field considered by Boyd, 1994). The meaning of the inequality $m^{2}<l^{2}$ is that $-\beta / m^{2}<U<0$, i.e. the translation speed of the current system determined by Eqs. (6) falls into the interval of phase speeds of Rossby waves (see, e.g. Kamenkovich et al., 1986). Correspondingly, in contrast to the LR modons, this high-smoothness solution damps slowly at infinity:

$$
\begin{gathered}
\psi \approx-\sqrt{\frac{2}{\pi \lambda r}}\left[B \cos \left(\lambda r-\frac{\pi}{4}\right)+C \sin \left(\lambda r-\frac{\pi}{4}\right)\right] \\
\text { at } r \rightarrow \infty
\end{gathered}
$$

thus, justifying its name "the nonlocal modon" suggested by Boyd (1994).

In the particular case of $m^{2}=l^{2}$, the localized highsmoothness solution to Eq. (4) is the Lamb modon:

$\psi= \begin{cases}-\left(\frac{2 U}{\kappa J_{2}\left(\kappa r_{0}\right)} J_{1}(\kappa r)+U r\right) \sin \theta, & r<r_{0} \\ -\frac{U r_{0}^{2}}{r} \sin \theta, & r>r_{0},\end{cases}$

where $U=U_{c r}=-\beta / m^{2}$ is the so-called Rossby critical ("long wave") speed. The "dispersion relationship" for this solution is $J_{1}\left(k r_{0}\right)$, i.e. $k r_{0}=j_{1, i}$, where $j_{1, i}$ is the $i^{\text {th }}$ root of the function $J_{1}$. Based on the results of KB (see also Orlandi et al., 1994), we may confine ourselves to the simplest dipolar solution corresponding to the first root of $J_{1}$, i.e. when $k r_{0}=j_{1,1} \approx 3.83$ (otherwise, the vortex is strongly unstable).

The Lamb modon on the $\beta$-plane $\left(m^{2}=l^{2}\right)$ separates the westward-propagating LR modons $\left(m^{2}>l^{2}\right)$ from the nonlocal modons $\left(m^{2}<l^{2}\right)$ given by Eq. (6) and provides a limiting case of both of them at $l^{2} \rightarrow m^{2} \pm 0$. To show this, let us first consider the westward-propagating LR modon Eqs. (5) (in which case, $l^{2}$ in Eqs. (4) and (5) bears a minus sign) and postulate that $m^{2}-l^{2}$ tends to zero from the right (so that $l^{2}$ tends to $m^{2}$ from the left). Since the functions $K_{1}(z)$ and $K_{2}(z)$ can be asymptotically represented as

$K_{1}(z) \approx \frac{1}{z}, \quad K_{2}(z) \approx \frac{2}{z^{2}} \quad$ at $z \rightarrow 0$,

one immediately obtains from Eqs. (5a) and (5c) that $\psi=$ $-\left(U r_{0}^{2} / r\right) \sin \theta$ at $r>r_{0}$. On the other hand, from Eqs. (5d) and (8) we have

$J_{1}\left(\kappa r_{0}\right) \approx-\frac{J_{2}\left(\kappa r_{0}\right)}{2 \kappa}\left(m^{2}-l^{2}\right) r_{0}$ at $m^{2}-l^{2} \rightarrow 0$,

(where again, $\kappa=\sqrt{k^{2}-m^{2}}$ ), i.e. $J_{1}\left(\kappa r_{0}\right)$ tends to zero, which is the "dispersion relationship" for the Lamb modon. According to Eqs. (9) and (5b), $A_{L} \rightarrow-2 U /\left[\kappa J_{2}\left(\kappa r_{0}\right)\right]$. Thus, the westward-propagating LR modon tends to the Lamb modon when $l^{2}$ approaches $m^{2}$ from the left. 
Now let us consider the nonlocal modon Eqs. (6) at $\mathrm{m}^{2}-$ $l^{2} \rightarrow 0-$, i.e. when $\lambda \rightarrow 0$. Suppose that the coefficient $A_{N}$ in Eq. (6a) is finite, i.e. $0<A_{N}<\infty$. By virtue of Eq. (6b), this implies: $J_{1}\left(\kappa r_{0}\right) \sim \lambda^{2} \rightarrow 0$. Using the following asymptotic representations of the functions $J_{1}(z), J_{2}(z)$, $N_{1}(z)$ and $N_{2}(z)$

$J_{1}(z) \approx \frac{z}{2}, \quad J_{2}(z) \approx \frac{z^{2}}{8}$,

$N_{1}(z) \approx-\frac{2}{\pi z}, \quad N_{2}(z) \approx-\frac{4}{\pi z^{2}} \quad$ at $z \rightarrow 0$,

one obtains from Eq. (6e) that $D \approx 2 \lambda /\left(\pi \kappa r_{0}\right)$. Hence, in view of Eqs. (10), (6c) and (6d), the terms that appear in the bottom row of Eq. (6a) for $r>r_{0}$ become asymptotically

$B_{N} J_{1}(\lambda r) \approx \frac{A_{N}}{4} E \pi \kappa r_{0} r$

$C_{N} N_{1}(\lambda r) \approx \frac{A_{N}}{2} \kappa J_{2}\left(\kappa r_{0}\right) \frac{r_{0}^{2}}{r}$

where $E=\lambda J_{2}\left(\kappa r_{0}\right) N_{1}\left(\lambda r_{0}\right)-\kappa J_{1}\left(\kappa r_{0}\right) N_{2}\left(\lambda r_{0}\right)$. According to Eqs. (11), $E$ must tend to zero in order for the solution (6) to be bounded at $r \rightarrow \infty$. This requirement, along with Eq. (10), implies that $J_{1}\left(\kappa r_{0}\right) \rightarrow \lambda^{2} r_{0} J_{2}\left(\kappa r_{0}\right) / 2 \kappa$ and, subsequently,

$A_{N} \rightarrow \frac{-2 U}{\kappa J_{2}\left(\kappa r_{0}\right)}, \quad B_{N} J_{1}(\lambda r) \rightarrow 0$

$C_{N} N_{1}(\lambda r) \approx-\frac{U r_{0}^{2}}{r}$

Due to Eqs. (12), the nonlocal modon tends to a Lamb modon if $l^{2} \rightarrow m^{2}+0$. Note that, in contrast with a LR modon that always tends to a Lamb modon at $l^{2} \rightarrow m^{2}-0$, two conditions must be applied to provide a transition from nonlocal modon to a Lamb modon: (i) the limiting solution must be bounded ( $A_{N}<\infty, E \rightarrow 0$ ), and (ii) it must be non-trivial $\left(0<A_{N}\right)$; otherwise, as seen from Eqs. (6), (10), if $A_{N} \rightarrow 0$, then the solution tends to a zonal flow $\psi=$ $\beta y / m^{2}$.

Westward-propagating modons are known to be unstable to tilt perturbations (Makino et al., 1981; Zabusky and McWilliams, 1982; Nycander, 1992; Hesthaven et al., 1993; Velasco Fuentes and Van Hejst, 1994; KB). In addition, the nonlocal modons, even when travelling exactly westwards, radiate Rossby waves (Fierl and Haines, 1994; KB). Nevertheless, in our preliminary numerical experiments (conducted at $m^{2}=0$ ), they died out slowly and exhibited quite a good survival in head-on interactions with LR modons (KB). Therefore, much more ambitious computations are needed to follow the evolution of the structures given by Eqs. (6) at $m^{2}=0$.

\section{Numerical scheme}

The calculations discussed below were based upon the numerical model quite similar to that described in KB. A rectangular basin $(-X \leq x \leq X,-Y \leq x \leq Y)$ with periodic boundary conditions at $x= \pm X$ was considered. The nondivergent and divergent versions of the model differed in the boundary conditions at $y= \pm Y$. The condition $\left.\psi\right|_{y= \pm Y}=0$ was applied in the nondivergent case, while in the divergent case, at every time step the constant values of stream function at the boundaries $y= \pm Y$ were computed from the boundary condition:

$\int_{-X}^{X} \frac{\partial^{2} \psi}{\partial t \partial y} d x=0$ at $y= \pm Y$,

which is valid for the periodic free-surface channel (Buchwald, 1973; Larichev, 1974; see also Kamenkovich et al., 1986).

Nondimensional finite-difference analogues of Eqs. (1) and (2) were used, the scales being $L=50 \mathrm{~km}$ and $T=$ $1 / \beta L \approx 10$ days for the space and time variables (synoptic length and time scales), and $\psi^{*}=\beta L^{3}$ and $q^{*}=\beta L$ for the stream function and PV, respectively. In the experiments presented below, the basin dimensions were $60 L \times 60 L$, the mesh size being $\delta=0.1 \mathrm{~L}$. In the course of the computations, the time step was controlled by the gradients of $\psi$ and $q$ and did not exceed $2.5 \times 10^{-3} T$. At every time step, PV and $q$ were computed from the discrete version of Eq. (2) first, and thereafter, the stream function, $\psi$, was found as a solution to the discrete analogue of the Helmholtz Equation (1). When computing $q$ for the current $t$, a combination of the direct and Matsuno schemes and Arakawa approximation for the Jacobian operator in Eq. (2) were adopted (Mezinger and Arakawa, 1976). The decomposition into eigenfunctions in the $x$-direction and the marching method in the $y$-direction (Samarsky, 1989) were utilized while solving Eq. (1). Conservation of total energy and enstrophy were found to hold within 0.1 to $0.3 \%$ in all our experiments.

In the following text, we use dimensional variables and constants, whereas in the figures and Table 1, all the quantities are nondimensional.

\section{Results and discussion}

\subsection{Evolution of a nondivergent, nonlocal modon}

Solution (6) can be characterized by three space scales: $r_{0}$, $1 / l$ and $1 / k$. The first is an overall scale; the second is the scale in the exterior area, where the contours of PV are open; and the third is the scale for the interior area, where the contours are closed (trapped fluid area). In order to make the rigid-lid condition valid and the $\beta$-effect significant in the balance of PV, these scales should be of the same order of magnitude. On the other hand, for the relationship (4) at $U<0$ to be nonlinear, i.e. for Eqs. (6) to represent a nonlinear solution, the parameters $l$ and $k$ must differ from each 

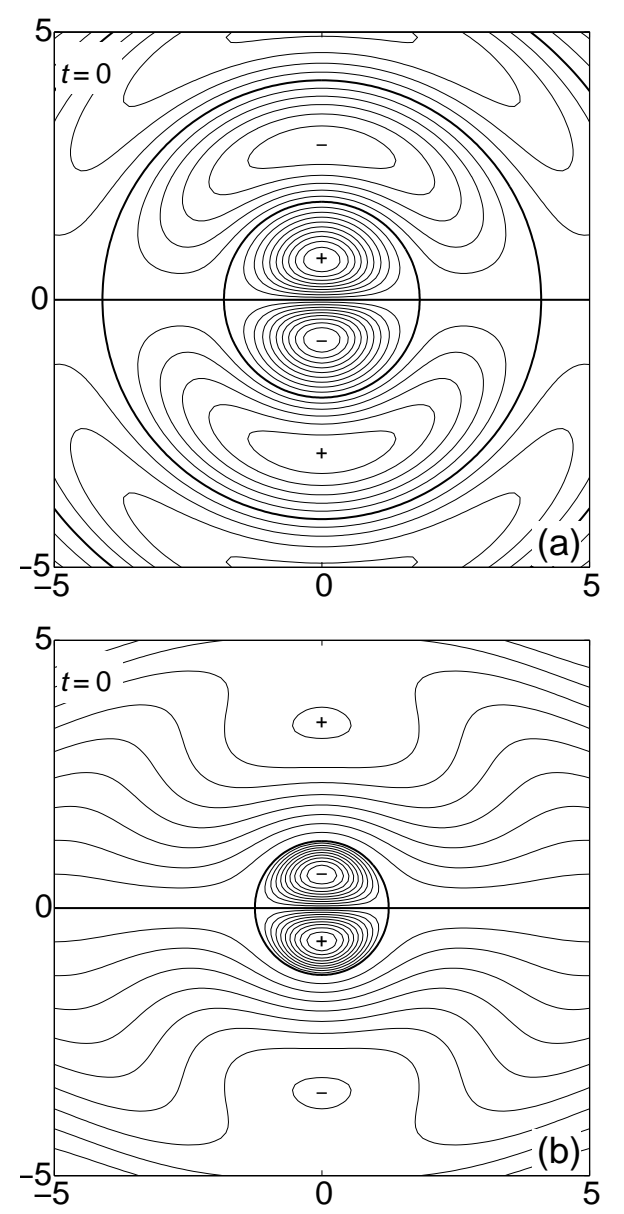

Fig. 1. The nondivergent, nonlocal modon in terms of stream function (a) and potential vorticity (b) (for parameters, see Table 1). The interval between the contours is $10 \%$ of the maximum.

other $(l<k)$, and the larger the ratio $k / l$ is, the stronger the nonlinearity of the solution. Based on this fact, the solutions in which the values of $l$ and $k$ are close in order of magnitude will be referred to below as moderately nonlinear.

A moderately nonlinear, nondivergent, nonlocal modon (NDNM) was chosen for the first evolutionary experiment (Figs. 1a, b and 2a, b); its nondimensional parameters are presented in Table 1. This NDNM displayed considerable robustness. Its dynamics can be roughly divided into two stages. At the first stage, until the time $t \approx 30 T$, no noticeable change in form was observed (Fig. 2a-d), with insignificant fluctuations of the translation speed being within the measurement error. During this period, the modon moved westward by about $15 \mathrm{~L}$, where the vorticity filament emission began (Fig. 2d). At the second stage (up to $t=50 T$ ), the filament emission grew stronger, while the main amount of PV was kept inside the vortical core around the poles (Figs. 2e, f). Since the interior area bounded by the zero $q$ contour must (formally) remain constant, to which a high degree of accuracy was held in all our experiments, the area of the vortex core decreased during this period (Figs. 2e, f).

Although the absolute value of the translation speed in-
Table 1. Nondimensional parameters of the nondivergent nonlocal modon (NDNM), divergent nonlocal modon (DNM), and the Lamb modon (LM) used for the initialization of the models in the evolutionary experiments

\begin{tabular}{lccccc}
\hline & $m^{2}$ & $r_{0}$ & $k^{2}$ & $l^{2}$ & $U=-\beta / l^{2}$ \\
\hline NDNM & 0 & 1.25 & 7.9942 & 2.0 & -0.5 \\
DNM & $m^{2}=$ & 1.50 & 8.6101 & 2.5 & -0.4 \\
& $0.9 l^{2}=2.25$ & & & & \\
LM & $m^{2}=l^{2}=2$ & 1.50 & 8.5252 & 2.0 & -0.5 \\
\hline
\end{tabular}

creased remarkably during the second stage of evolution, the changes were relatively slow, with the characteristic time of this positive trend in $|U|$ being much longer than the time scale $T$ (Figs. 3a, e). This fact allows for the treatment of the evolving vortex as quasi-stationary at every $t$. We approximated the vortex by an exact solution of the type (6) for the times $t=n T, n=1, \ldots, 50$. This fitting has proved that, in spite of the filamentation and a very slight ellipticity of the vortical core, the vortex structure, while evolving, keeps its generic features, i.e. it remains of the NDNM type, with the zero $q$ contour (that bounding the core) being essentially circular everywhere aside from the filament area.

In fitting an exact solution to an instantaneous $\psi$ field, we followed the procedure containing the following steps (for details, see KB). For a fixed $t$, the radius of the nearly circular core, $r_{0}(t)$, could be defined as the mean radius of zero contour of either the PV field or $\Psi=\psi+U y$ field. The zero contours for these fields are identical if the functional dependence (4) is held exactly. However, they differ slightly from each other: the $\Psi$ field is always smoother (has no filaments) and is less elliptical. Therefore, in our experiments, we evaluated $r_{0}$ by analyzing the $\Psi$ field. The other two parameters describing NDNM are the two coefficients of proportionality, $k^{2}$ and $l^{2}$, in the $q$ vs. $\Psi$ relation (4). They were obtained from the scatter-graphs $q$ vs. $\Psi$ (see, e.g. Fig. 4). Notice that $l^{2}$ determined in such a way must be close to that determined as $l^{2}=-\beta / U$, and this was the case in our experiment. This fact allowed for the use of the instantaneous translation speed $U$ as the third parameter (instead of $l^{2}$ ), along with $k^{2}$ and $r_{0}$, to identify the vortex in terms of NDNM at any $t$.

The goodness-of-fit can be seen from the comparison of the cross sections of the computed and fitted theoretical fields of $\psi$ and $q$ at $t=50 T$ (Fig. 5). The main difference is observed far away from the core center and can be explained as a boundary effect. Indeed, the exact solution tends slowly to zero at $r \rightarrow \infty$, while the computed solution satisfies zero $\psi$ conditions at the northern and the southern boundaries (this discrepancy motivated us to consider a relatively large grid square - see Sect. 3).

The time evolution of the main vortex parameters $r_{0}, k$ and $U$ (estimated through the fitting) is shown in Figs. 3ce. The changes in all the parameters by $t=30$ were insignificant (Figs. 3b-f). In the subsequent evolution, the vortex contracted considerably, its radius and the interior stream 

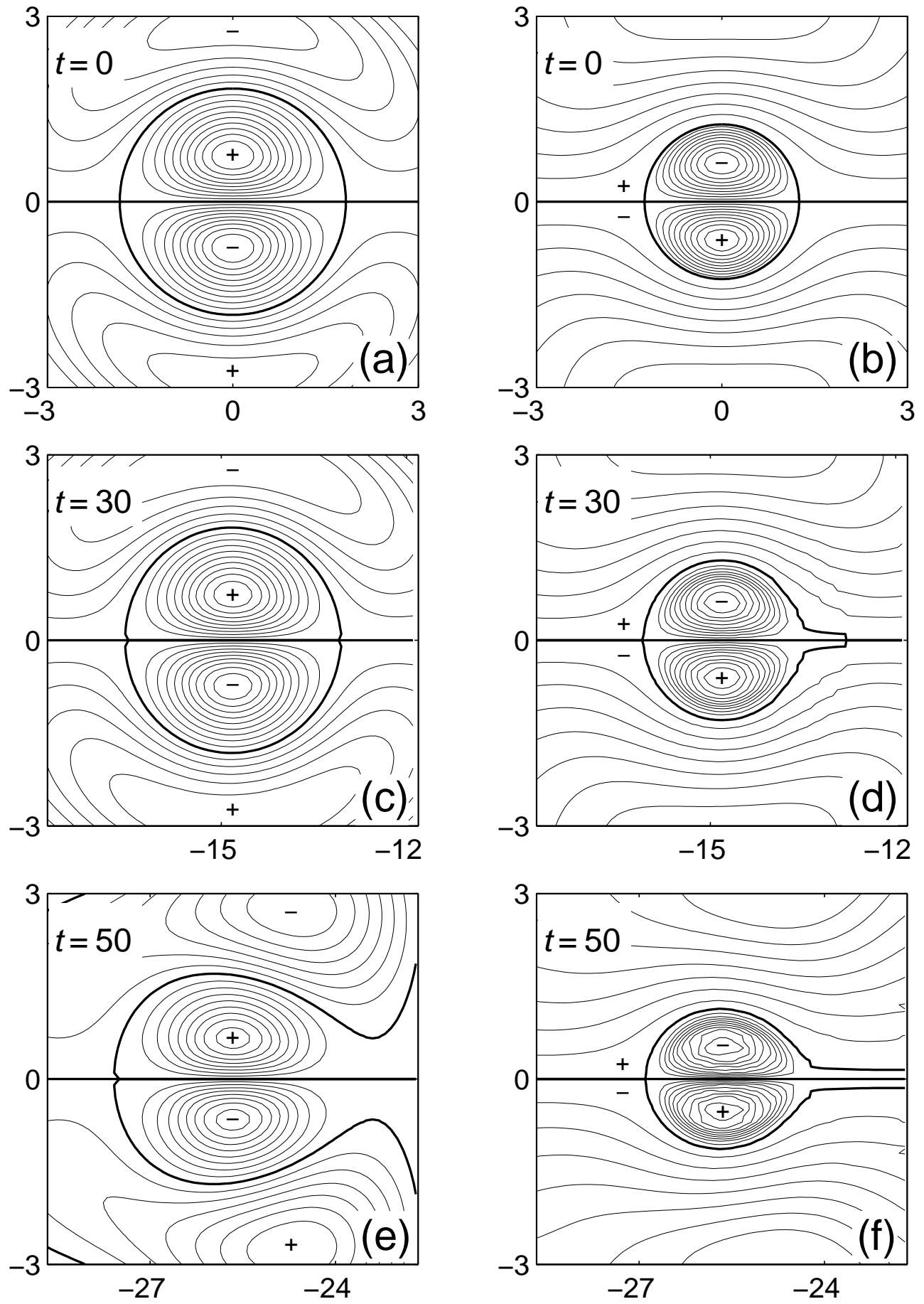

Fig. 2. Long-term evolution of the nondivergent, nonlocal modon in terms of stream function (on the left - (a), (c), (e)) and potential vorticity (on the right - (b), (d), (f)). The interval between the contours is $10 \%$ of the initial maximum.

function peak decreased (Figs. 3b, c), while the translation speed increased in absolute value (Fig. 3e). Nonlinearity of the NDNM (in the above sense) increased as well, because, due to the speeding up of the modon, the parameters $l=\sqrt{-\beta / U}$ and $k$ decreased and increased, respectively, (Fig. 3d). The decrease in $l$ also means that the enhancement of the translation speed is attended with an increase in the far field "wavelength" and thus, with some smoothing down of this field.
As explained above, the filament emission must result in the decrease of the vortex radius, since, due to the PV conservation, the area inside a certain closed PV contour must remain constant. In other words, at the second stage of evolution, the vortex core contracts (Figs. 2c, f and 3c), the points corresponding to the peak PV values become closer, and the nonlinearity ( $k$ to $l$ ratio) increases, which leads to sharpening of the radial PV gradients in the interior. Other quantities that must be conserved are the peak values of $q$. It is worth 

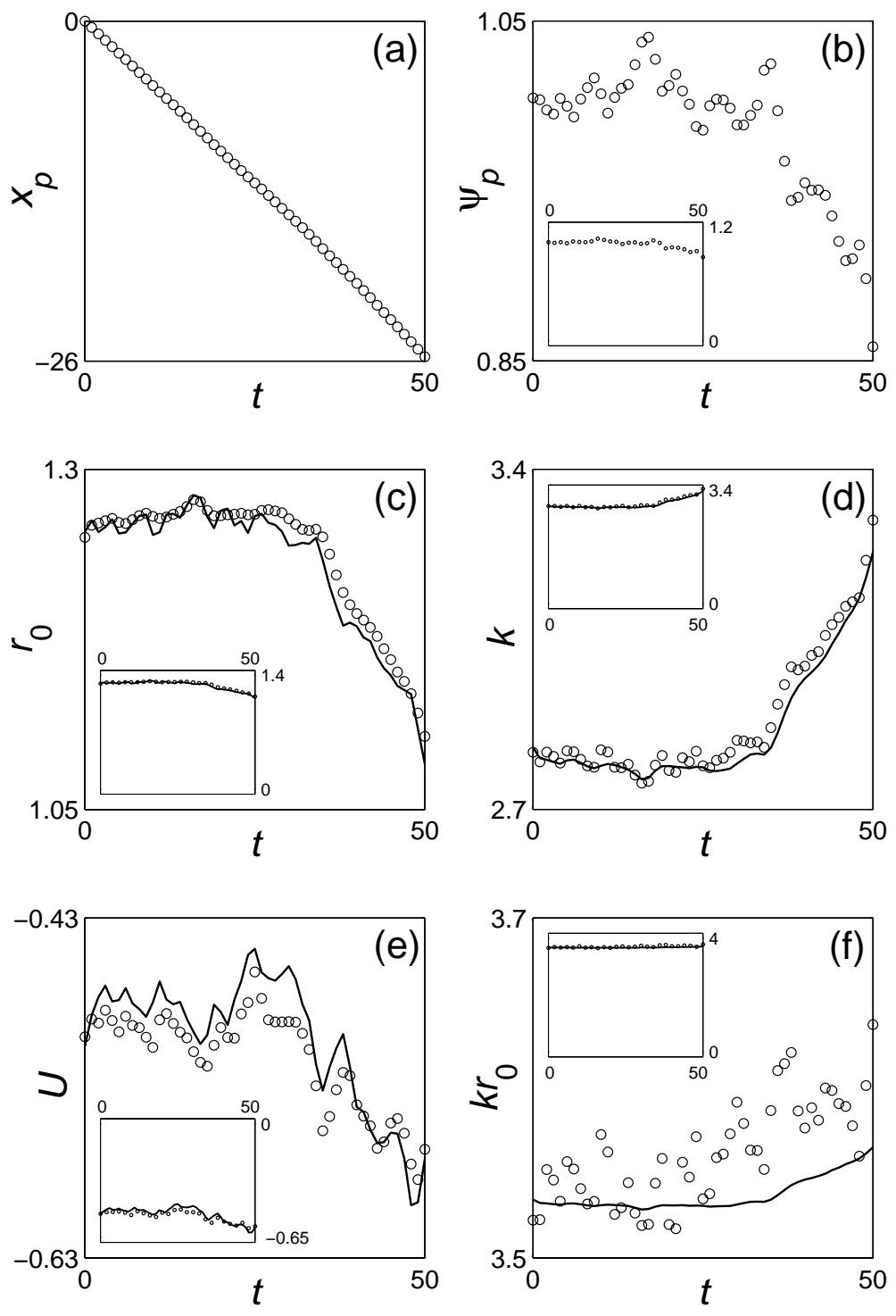

Fig. 3. Evolution of the parameters of the nondivergent, nonlocal modon. Circles - pure experimental data, solid lines - data computed via Eqs. (13), large panels - close-up ranges of the variables, insets - full-scale plots including the origin:

(a) $-x$-coordinate of the vortex peaks; (b) - the peak value of stream function; (c) - the radius of the vortex core, $r_{0}$; (d) - square root of the factor of proportionality between internal $q$ and $\Psi$ in Eq. (4), $k$;

(e) - the translation speed of the vortex, $U$;

(f) - parameter $k r_{0}$.
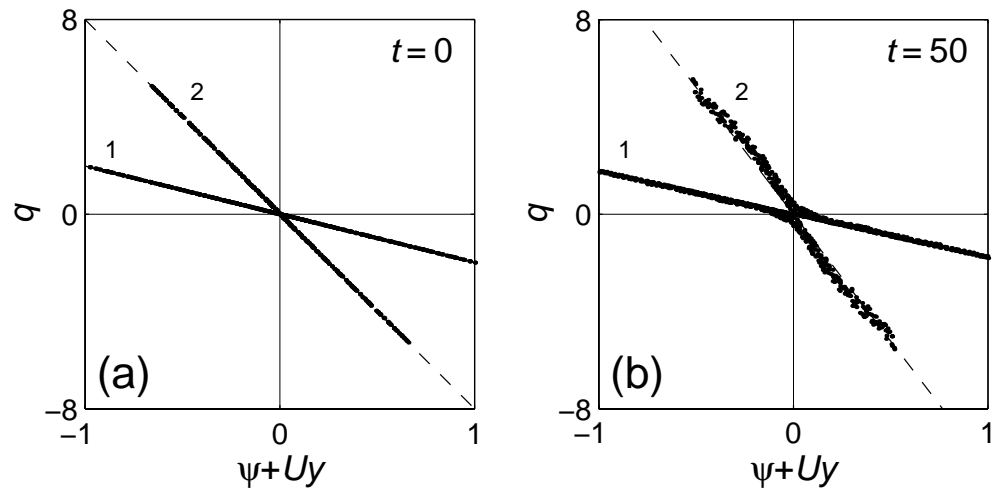

Fig. 4. Initial (a) and final (b) scatter diagrams of $q$ vs. $\Psi=\psi+U y$ for the nondivergent, nonlocal modon: external (1) and internal (2) regions.

noting that the filamentation took place due to the emission of fluid particles bearing quite small absolute values of PV (Fig. 7). This is why the vortex core kept its dipolar shape (the peak PV values being the poles in terms of isolines of $q$ ).
In the described experiment, due to numerical effects, the peak absolute values of PV increased slightly at the stage of filament emission. Our simulation lasted while this deviation from the initial peak value remained insignificant and 

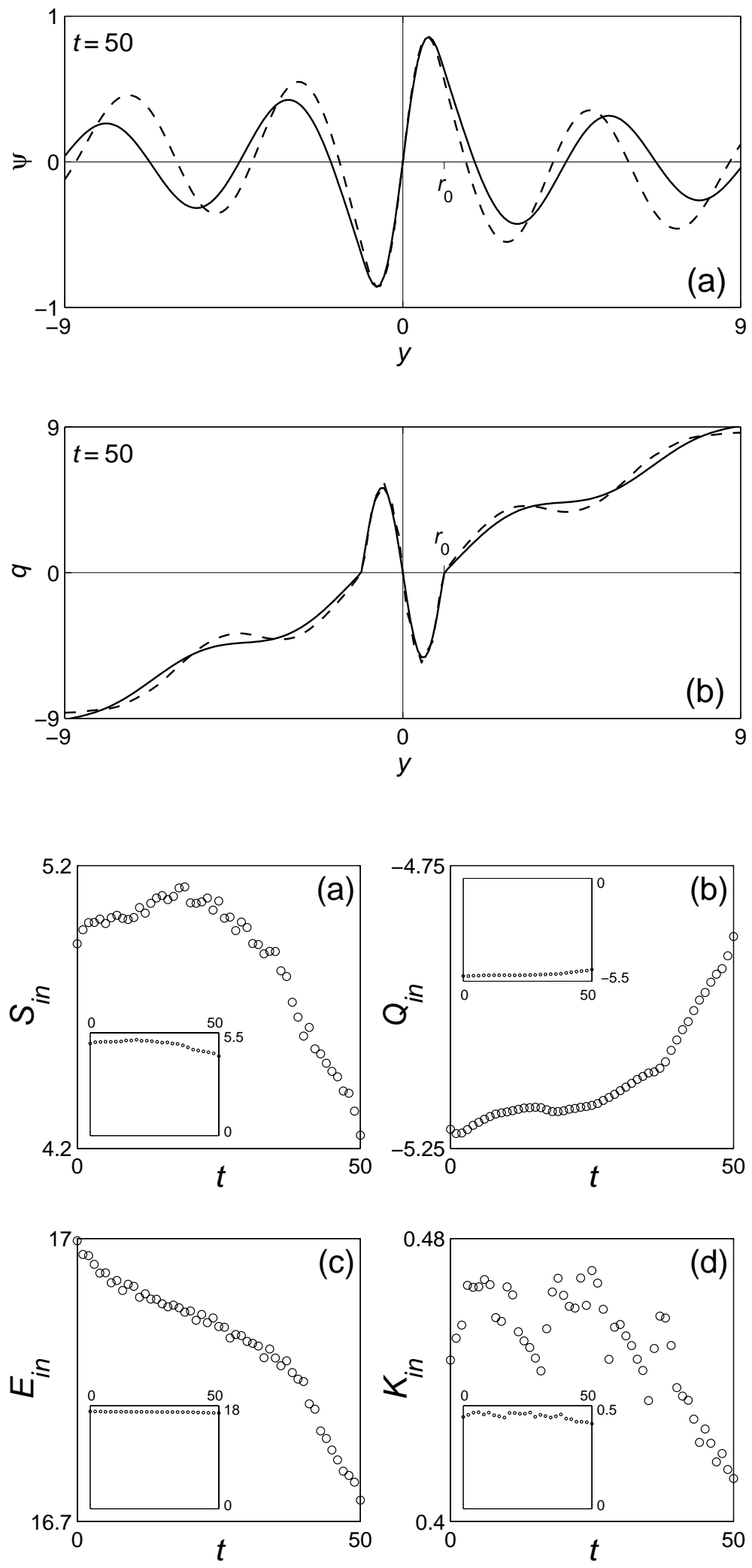

Fig. 5. Fitting the vortex evolved from the nondivergent, nonlocal modon in terms of stream function (a) and potential vorticity (b). Solid lines - experimental data; dashed lines - fitted theoretical modon.

Fig. 6. Evolution of the integral parameters of the nondivergent, nonlocal modon:

(a) - core area;

(b) - potential vorticity (integrated within the upper half of the core);

(c) - core enstrophy;

(d) - core kinetic energy (large panels close-up ranges of the variables, insets - full-scale plots including the origin).

the computations were stopped at $t=50 \mathrm{~T}$, when the deviation reached approximately the limit of $2 \%$ (see below). (We attribute this small numerical error to the adverse influence of the boundaries (due to the non-locality of the vortex), as well as to the constantly increasing nonlinearity of the vortex during the contraction phase or, more precisely, to the increase in the radial $q$ gradients in the interior: at the first phase the error was extremely small.) Formally speaking, 

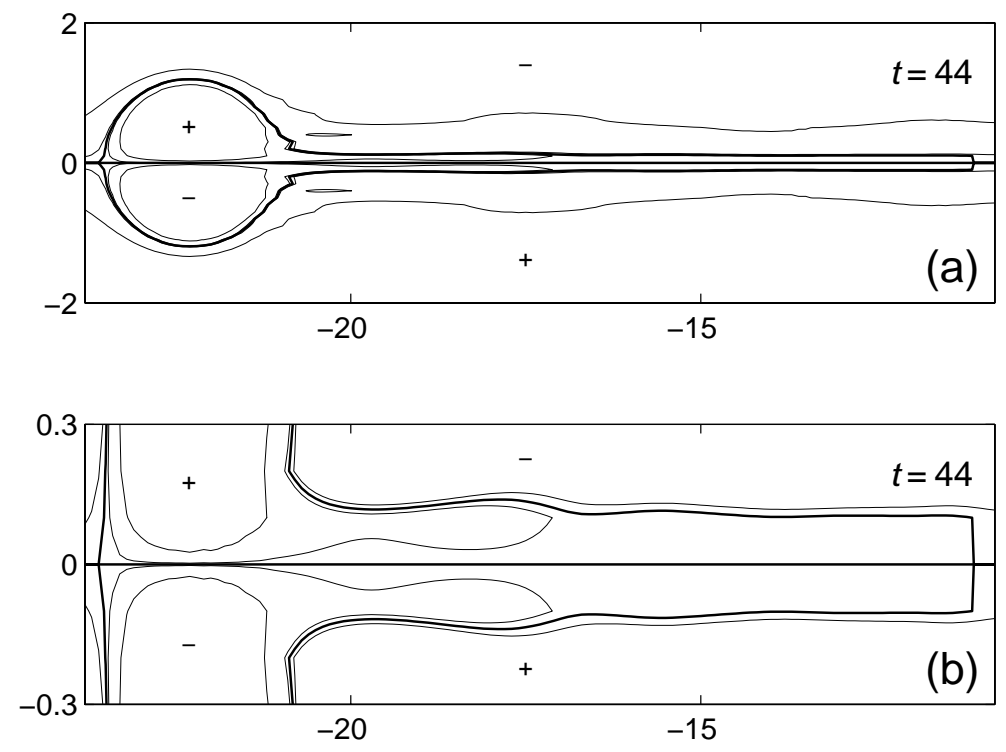

Fig. 7. Structure of the vorticity filament emitted by the nondivergent, nonlocal modon. The contours correspond to 0,1 , and 10 percent of the peak potential vorticity value (upper panel equal scales in the $x$ - and $y$-directions, lower panel - close-up in the $y$-axis direction).

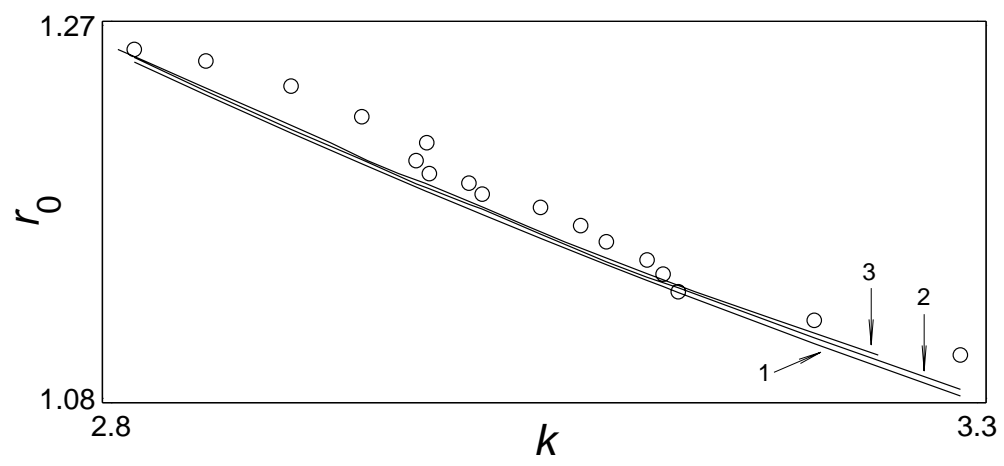

Fig. 8. Dependence of the core radius on $k$ for the evolving nondivergent, nonlocal modon.

Circles - "raw" experimental data; solid lines - estimated via Eqs. (13) using different peak values of the potential vorticity:

1 - initial,

2 - final,

3 - changing-in-time raw values.

since the peak PV values of the vortex core must be conserved during the vortex contraction, the peaks of the relative vorticity $(\Delta \psi=q-\beta y)$ not only become closer, but increase in their absolute value. This leads to a more intensive interaction between the dipole halves and results in the acceleration of the westward drift of the vortex pair (Fig. 3e). Due to the fact that $k$ increases and $r_{0}$ decreases within the period $30 T<t<50 T$, the product $k r_{0}$ changes quite slowly, demonstrating a tendency to increase (Fig. 3f). However, the limit value $j_{1,1} \approx 3.83$ (see Eq. 7) cannot be reached while the vortex remains of the NDNM type.

The evolution of the integral characteristics of the vortical core is shown in Fig. 6. The core area, enstrophy and kinetic energy were computed by the integration within the zero $q$ contour, with the filaments being cut off. The integral PV shown in Fig. 6b corresponds to the upper half of the core (since due to the antisymmetry the integral PV calculated over the vortex pair equals zero). Throughout the experiment, the change of the total enstrophy was small but monotonic (Fig. 6c): the two stages of the evolution can be distinguished only through the difference in the slopes of this graph by $t=30 T$ and within the period $30 T<t<50 T$. However, in the graphs of the core area, integral PV, and the kinetic energy versus time (Figs. 6a, b, d), the two stages are clearly seen: before the strong filament emission takes place, $S_{i n}, Q_{i n}$ and $K_{i n}$ hardly change, whereas at the filamentation stage, they decrease considerably. The loss in $Q_{i n}$ and $K_{i n}$ is about $8 \%$ and $14 \%$, respectively; the loss in $S_{i n}$ is the most impressive at about $18 \%$. Thus, during the second stage of the evolution, a considerable part of the vortex area and PV is transferred into a continuously elongating thin filament (Fig. 7).

The question arises: is the $2 \%$ computational error in the peak $q$ value indeed, negligible or can it significantly affect the apparent evolution of the vortex? To answer this question the following computations were carried out.

Assume that in the course of the evolution the vortex core and its close periphery (apart from the filament region - see Fig. 7) remain those of the NDNM type. Suppose next that the peak $q$ value does not change in the time remaining equal to the initial one (theoretically, this peak value must be constant). Finally, let the stream function peak value and $y$ coordinate of this peak be given as functions of time. Then based on these data we can compute the parameters $r_{0}, k^{2}$ and $U$ as functions of time by solving the following system 

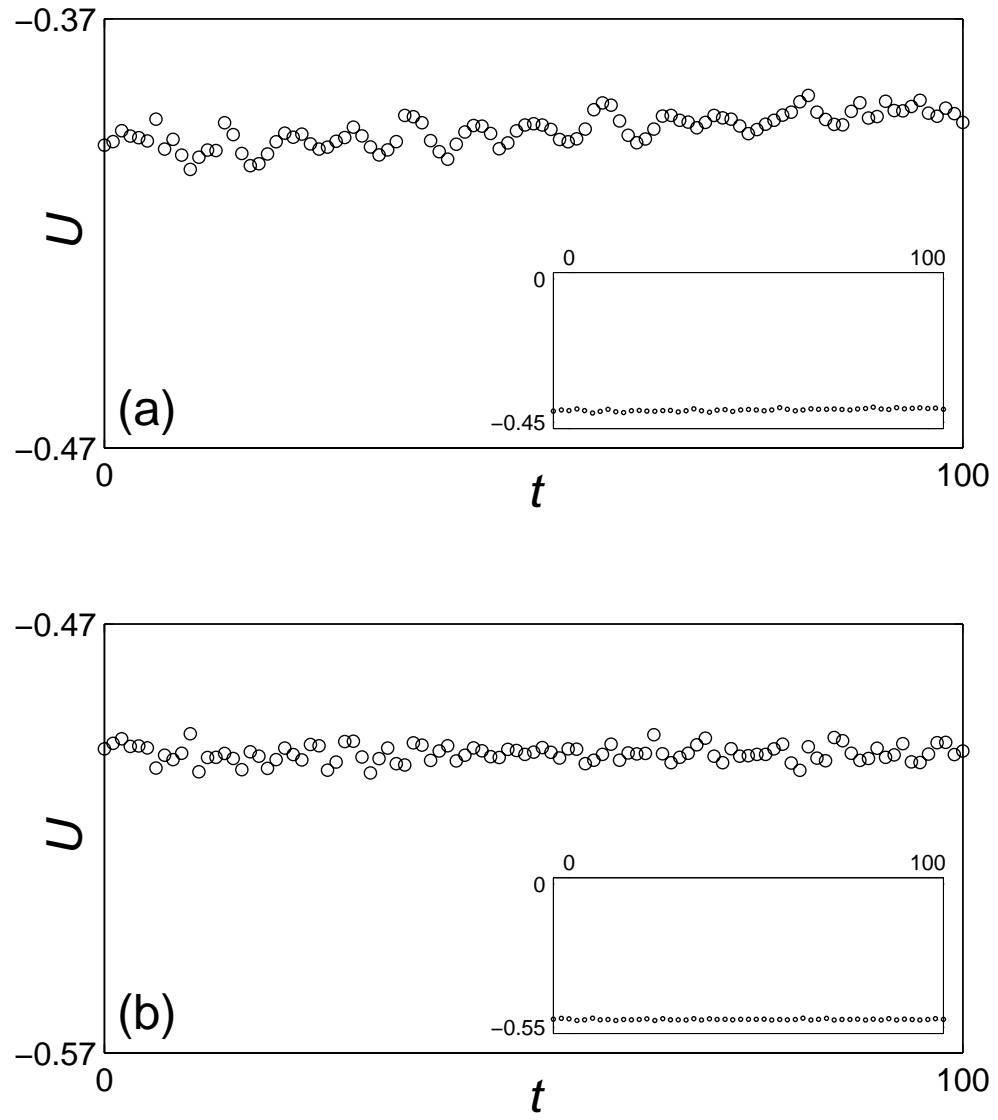

Fig. 9. Experimental translation speeds of the divergent nonlocal modon (a) and the Lamb modon (b) (for initial parameters, see Table 1; large panels - closeup ranges of $U$, insets - full-scale plots including the origin).

of equations that results from Eq. (6):

$$
\begin{aligned}
& \psi_{p}=\frac{\beta r_{0}}{k^{2}} \frac{J_{1}\left(k y_{\psi}\right)}{J_{1}\left(k r_{0}\right)}-\left(\frac{\beta}{k^{2}}+U\right) y \psi, \\
&-\beta r_{0}\left[J_{1}\left(k y_{\psi}\right)-k y_{\psi} J_{2}\left(k y_{\psi}\right)\right] \\
&+\left(\beta+k^{2} U\right) y_{\psi} J_{1}\left(k r_{0}\right)=0, \\
& q_{p}= \beta\left[y_{p}-r_{0} \frac{J_{1}\left(k y_{q}\right)}{J_{1}\left(k r_{0}\right)}\right], \\
& r_{0}\left(J_{1}\left(k y_{q}\right)-k y_{q} J_{2}\left(k y_{q}\right)\right)-y_{q} J_{1}\left(k r_{0}\right)=0 .
\end{aligned}
$$

Here, $\psi_{p}$ and $q_{p}$ are the given peak values of the stream function and PV, respectively, while $y_{\psi}$ and $y_{q}$ are the $y$ coordinates of these peaks. The first equation relates the $\psi$ peak value to its coordinate. The second equation can be obtained by differentiating Eq. (6a) at $r<r_{0}$, and states that the $y$-derivative of the stream function at the peak is zero. The third equation, which is obtained from the dependence (4) of $q$ upon $\psi+U y$ for the interior area, expresses the PV peak value as a function of its coordinate. The fourth equation (analogous to the second one) means that the $y$ derivative of $q$ at the peak point is zero. Given the parameters $q_{p}=\left.q_{p}\right|_{t=0}=$ const., $\psi_{p}(t)$ and $y_{\psi}(t)$, the system (13) can be solved to provide the values of the remaining variables $r_{0}$, $k, U$ and $y_{q}$ as functions of time.

As noted above, in the practical computations, the peak $q$ value does not remain constant, with the error being about
$2 \%$. On the other hand, as the stream function field is determined from the second-order Eq. (1), it is much smoother than the PV field and less distorted by computational errors. For this reason, it is legitimate to use the experimental values $\psi_{p}(t)$ and $y_{\psi}(t)$ (along with initial $q_{p}$ ) to estimate the "theoretical" parameters $r_{0}, k$ and $U$. These estimates are shown in Figs. 3c- $\mathrm{f}$ by continuous lines. The closeness of the "theoreticaly" estimated graphs to the "experimental" points testifies to (i) the reliability of our simulations, and (ii) the consistency of the assumption that the vortex remains essentially of the NDNM type while contracting and speeding up.

In addition to the above calculations based on the given $q_{p}=\left.q_{p}\right|_{t=0}=$ const., we also calculated the "theoretical" estimates of $r_{0}$ and $k$ using both the peak $q$ values observed during the simulation and based on the final peak value $\left(q_{p}=\left.q_{p}\right|_{t=50 T}=\right.$ const. $)$. These computations represent additional evidence of the reliability of our simulation and its interpretation. As seen from Fig. 8, where the $r_{0}$ versus $k$ scatter (raw experimental data) is shown, along with the three kinds of "theoretically" estimated dependencies, the four trends are very close to each other.

In the described experiment, no pronounced tendency for minimization of the far field (similar to that predicted by the theory of weakly nonlocal solitary waves, Boyd, 1998a, b) was observed. One might suppose that the duration of the simulation $(t=50 T)$ was insufficient for the vortex to reach 

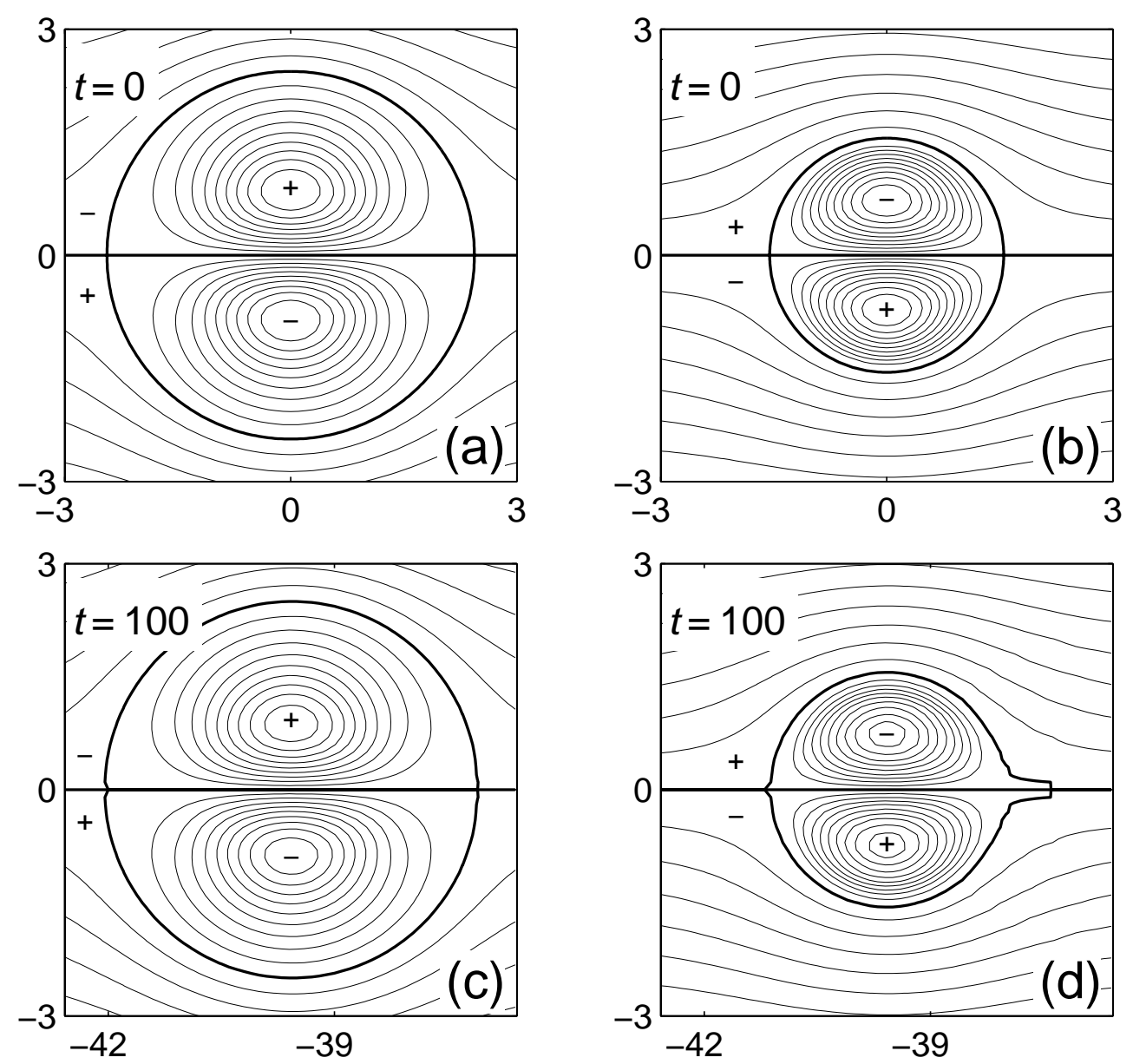

Fig. 10. Evolution of the divergent nonlocal modon in terms of stream function (on the left - (a), (c)) and potential vorticity (on the right (b), (d)). Contours - as in Fig. 2.

such a steady state. We, however, are inclined to believe that for the nonlocal modon solutions of the equation of PV conservation on the $\beta$-plane, this issue is less topical than for the nonlocal phenomena considered by Boyd (1998a, b). Most likely, due to the filamentation, which becomes significant by $t=50 T$, and the related core contraction, the modon will essentially die out at the earlier times as compared to that needed for the far field minimization.

\subsection{Evolution of a divergent, nonlocal modon}

As discussed above, during the filament emission process, the product $k r_{0}$ in NDNM increases being bounded by the limit $k r_{0}=j_{1,1}$. The upper limit, however, cannot be achieved because under the rigid-lid condition, there is no westward-propagating solution to Eq. (4) that obeys this "dispersion relationship". However, in the framework of the divergent model, such a solution does exist. It is the Lamb modon (7), which corresponds to the case of $m^{2}=l^{2}$. We carried out an evolutionary experiment with a divergent nonlocal modon (DNM) that initially was close to this case $\left(m^{2}=0.9 l^{2}\right)$, in order to check out whether during the filamentation and speeding up of such a DNM can really ap- proach a Lamb modon. The nondimensional parameters of the DNM used in this experiment are given in Table 1: the DNM chosen for the experiment was of moderate nonlinearity, while its initial radius and translation speed were close to those of NDNM.

Although this simulation lasted for a period of time twice as long as that with the NDNM - up to $100 T$, no considerable increase in the translation speed was detected (Fig. 9a). Moreover, throughout the experiment, the changes in the form of the vortex were very slight, not only in the vortex core but in the vortex periphery as well (Fig. 10). Very weak filament emission appeared only at large times (Fig. 10d). The DNM proved to be very robust, and no tendency of transition into a Lamb modon was observed. The computations were stopped at $t=100 T$, when the deviation in the $q$ peak value from the initial one reached approximately $2 \%$.

\subsection{Evolution of the Lamb modon}

A moderately nonlinear lamb modon (LM), with the initial radius and translation speed close to those of the NDNM and the DNM treated above, was chosen for testing in an evolutionary experiment (for parameters, see Table 1). 

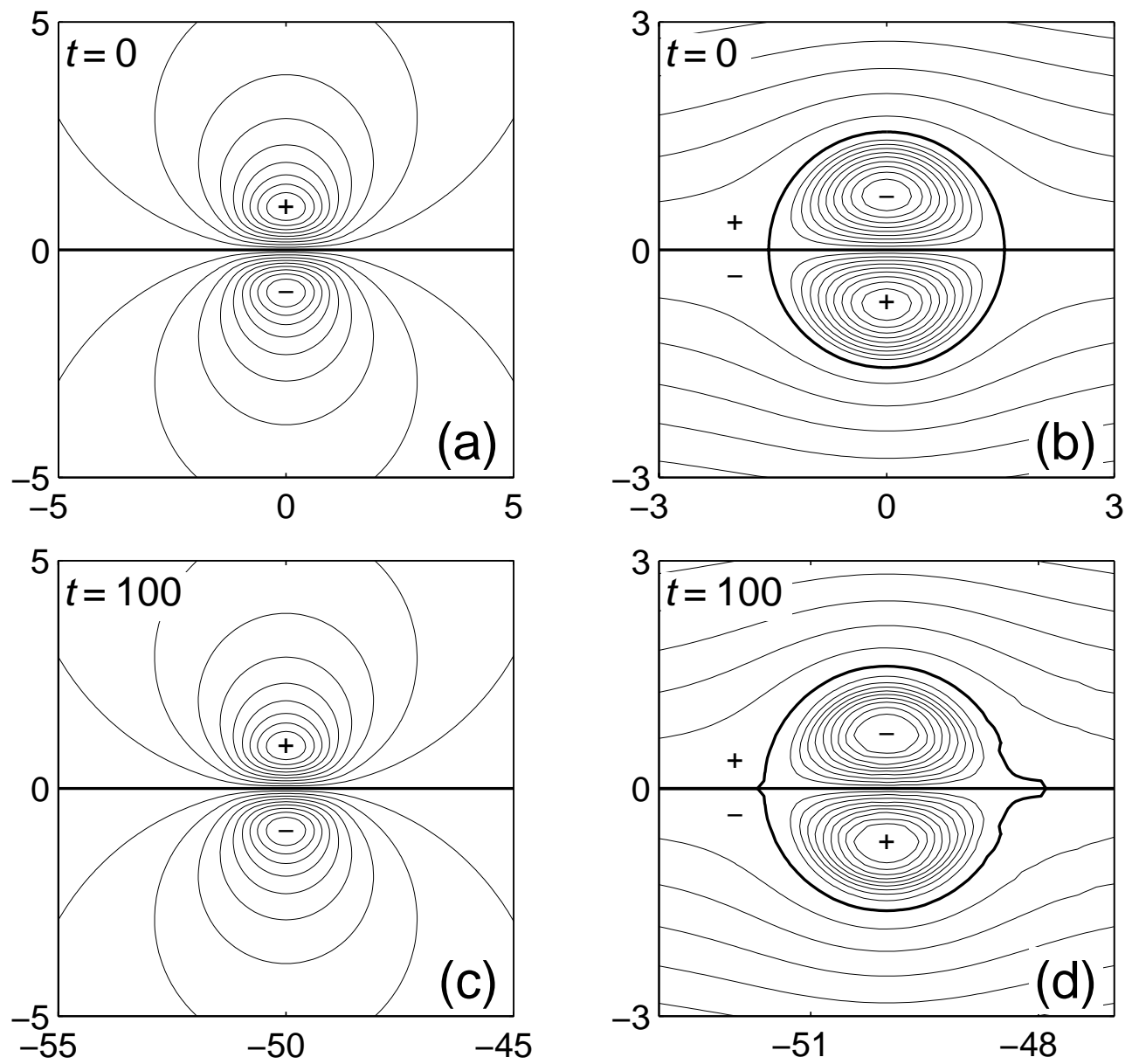

Fig. 11. Evolution of the Lamb modon in terms of stream function (on the left - (a), (c)) and potential vorticity (on the right - (b), (d)). Contours - as in Fig. 2.

During this run that lasted for $100 T$, the translation speed of the LM actually remained constant (Fig. 9b), whereas the modon hardly changed its form (Fig. 11), demonstrating no tendency to evolve into a westward-propagating LR modon. Filamentation started only a short time before the end of the experiment and was extremely weak. The LM modon proved to be the most persistent of the three vortices examined in the present study, which is in good agreement with the numerical experiments performed by van Geffen and van Heijst (1998) and Babkin et al. (2000) on the $f$-plane.

\section{Conclusion}

Our simulations show that there are two stages in the longterm evolution of a nondivergent, nonlocal modon on the $\beta$ plane. The first robust stage $(t \leq 30 T)$, characterized by the fact that the vortex parameters and form change insignificantly, is in correspondence with the numerical evolutionary experiments carried out by Flierl and Haines (1994) in the case of a barotropic model under the rigid-lid and weak $\beta$ effect conditions initialized by a westward-travelling Lamb modon. At the second stage, the emission of vorticity fil- aments occurs, resulting in a considerable contraction and speeding up of the vortex core. In spite of these changes, the vortex remains essentially of the nonlocal modon type. These results are in agreement with the laboratory experiments of Velasko Fuentes and van Heijst (1994), who found that as long as a dipole drifts westwards, it maintains approximately constant translation speed, while its size decreases. We attribute the constancy of the speed in their experiments to the balance between two effects: the above-described acceleration due to the contraction and the deceleration due to viscosity.

The divergent (equivalent-barotropic) nonlocal modons are, in general, more robust. More precisely, the closer the modon translation speed is to the critical (long wave) speed $U_{c r}=-\beta / m^{2}$, the longer the form-preserving span of the modon. For example, the nonlocal modon, whose initial propagation speed equals $0.9 U_{c r}$, persists for about $100 \mathrm{~T}$ without significant change in form and propagation speed, with the subsequent filamentation being much weaker than that of the nonlocal modon in the nondivergent model. The Lamb modon, which is the limiting case of both westwardtravelling LR and divergent nonlocal modons on the $\beta$-plane at $l^{2} \rightarrow m^{2}$ (i.e. when the translation speed tends to the 
critical one) proved to be the most persistent in our experiments.

Even though the initial translation speed of the divergent nonlocal modon in our experiment was very close to that of the Lamb modon, the vortex pair did not speed up during the simulation, i.e. no tendency of this modon to transform into a Lamb modon was observed. Similarly, the Lamb modon did not demonstrate any tendency to accelerate (i.e. to transform into a LR modon).

It should be mentioned that in our previous work (KB), several simulations with initially tilted (within a $5^{\circ}$ angle) nonlocal modons were considered. Despite the fact that the westward-propagating modons on the $\beta$-plane are known to be unstable to tilt perturbations (see Introduction), nonlocal modons usually kept the westward component of their drift for $10 T$ to $25 T$ remaining coherent dipoles (especially if a small tilt angle was applied to a slowly propagating nonlocal modon). Thereafter, they turned to the east (transforming into eastward-travelling LR modons) or disintegrated.

The results of our numerical experiments signify that nonlocal modons may present a good candidate for the cause of the atmospheric blocking phenomenon. They also suggest that the abundance of paired vortices in the ocean and in the atmosphere is due to both localized and nonlocal modons.

Acknowledgement. This research was supported by The Israel Science Foundation (grant \# 616/00). We also thank the anonymous referees for their helpful comments on the manuscript.

\section{References}

Ahnlas, K., Royer, T. C., and George, T. H.: Multiple dipole eddies in Alaska Coastal Current detected Landsat Thematic Mapper data, J. Geophys. Res., 92, 13 041-13 047, 1987.

Babkin, E. A., Brailovskaya, V. A., Clamond, D., Fraunie, Ph., and Stepanyants, Yu.: Evolution of concentrated vortices in a viscose fluid, IJCFD, 13, 185-202, 2000.

Berestov A. L.: Solitary Rossby waves, Izv. Akad. Sci. USSR, Atmos. Oceanic Phys., 15, 648-651, 1979.

Berestov A. L.: Some new solutions for the Rossby solitons, Izv. Akad. Sci. USSR, Atmos. Oceanic Phys., 17, 82-87, 1981.

Boyd, J. P.: Nonlocal modons on the beta-plane, Geophys. Astrophys. Fluid Dyn., 75, 163-182, 1994.

Boyd, J. P.: Radiative decay of weakly nonlocal solitary waves, Wave Motion, 27, 211-221, 1998a.

Boyd, J. P.: Weakly nonlocal solitary waves and beyond-all-orders asymptotics, Kluwer, 608 pp, 1998 b.

Buchwald, V. T.: Long period divergent planetary waves, Geophys. Fluid Dyn., 5, 359-367, 1973.

Butchart, N., Haines, K., and Marshall, J. C.: A theoretical and diagnostic study of solitary waves and atmospheric blocking, J. Atmos. Sci., 46, 2063-2078, 1989.

Couder, Y. and Basdevant, C.: Experimental and numerical study of vortex couples in two-dimensional flows, J. Fluid Mech., 173, 225-251, 1986.

DiBatista, M. and Polvani, L. M.: Barotropic vortex pairs on a rotating sphere, J. Fluid Mech., 358, 107-133, 1998.

Fedorov, K. N. and Ginsburg, A. I.: Mushroom-like currents (vortex dipoles): One of the most wide-spread forms of non-stationary coherent motions in the ocean, In: Mesoscale/Synoptic Coherent Structures in Geophysical Turbulence, (Eds) Nihoul, J. C. H. and Jamart, B. M., Elsevier, 1-14, 1989.

Flierl, G. R. and Haines, K.: The decay of modons due to Rossby wave radiation, Phys. Fluids, 6A, 3487-3497, 1994.

Flierl, G. R., Larichev, V. D., McWilliams, J. C., and Reznik, G. M.: The dynamics of baroclinic and barotropic solitary eddies, Dyn. Atmos. Oceans, 5, 1-41, 1980.

Flierl, G. R., Stern, M. E., and Whitehead, J. A.: The physical significance of modons: laboratory experiments and general integral constraints, Dyn. Atmos. Oceans, 7, 233-264, 1983.

Flór, J. B. and van Heijst, G. J. F.: An experimental study of dipolar vortex structures in a stratified fluid, J. Fluid Mech., 279, 101133, 1994.

Ginsburg, A. I. and Fedorov, K. N.: Mushroom currents in the ocean, Issledovania Zemli iz Kosmosa, 3, 18-26, 1984.

Haines, K.: Baroclinic modons as prototypes of atmospheric blocking, J. Atmos. Sci., 46, 3202-3218, 1989.

Haines, K. and Marshall, J. C.: Eddy forced coherent structures as a prototype of atmospheric blocking, Quart. J. Roy. Meteor. Soc., 113, 681-704, 1987.

Haupt, S. E., McWilliams, J. C., and Tribia, J. J.: Modons in shear flow, J. Atmos. Sci., 50, 1181-1198, 1993.

Hesthaven, J. S., Lynov, J. P., and Nycander, J.: Dynamics of nonstationary dipole vortices, Phys. Fluids, A5, 622-629, 1993.

Hooker, S. B., Brown, J. W., Kirwan, Jr., A. D., Lindemann, G. L., and Mied, R. P.: Kinematics of a warm-core dipole ring, J. Geophys. Res., 100, 24 797-24 809, 1995.

Ikeda, M. and Emery, W. J.: Satellite observations and modelling of meanders in the California Current system off Oregon and Nothern California, J. Phys. Oceanogr., 14, 1434-1450, 1985.

Ikeda, M., Mysak, L. A., and Emery, W. J.: Observation and modelling of satellite-sensed meanders and eddies off Vancouver Island, J. Phys. Oceanogr., 14, 3-20, 1984.

Johannessen, J. A., Svendsen, E., Sandven, S., Johannessen, O. M., and Legree, K.: Three-dimensional mesoscale eddies in the Norwegian Coastal Current, J. Phys. Oceanogr., 19, 3-19, 1989.

Kamenkovich, V. M., Koshlyakov, M. N., and Monin, A. S.: Synoptic eddies in the Ocean, Reidel, The Netherlands, 1986.

Kizner, Z. I.: Rossby solitons with axisymmetric baroclinic modes, Doklady Akad. Nauk USSR, 275, 1495-1498, 1984.

Kizner, Z. I.: Intense synoptic eddies and the quasigeostrophic approximation, Oceanology, 26, 16-20, 1986a.

Kizner, Z. I.: Strongly nonlinear baroclinic solitary Rossby waves, Oceanology, 26, 284-289, 1986b.

Kizner, Z. I.: On the theory of intrathermocline eddies, Doklady Akad. Nauk USSR, 300, 453-457, 1988.

Kizner, Z. I.: Solitary Rossby waves with baroclinic modes, J. Mar. Res., 55, 671-685, 1997.

Kizner, Z. and Berson, D.: Emergence of modons from collapsing vortex structures on $\beta$ - plane, J. Mar. Res., 58, 375-403, 2000.

Lamb, H.: Hydrodynamics, 6th ed. Dover, New York, NY, pp. 739, 1932.

Larichev, V. D.: The internal boundary problem for the Rossby wave equation, Izv. Akad. Sci. USSR, Atmos. Oceanic Phys., 10, 763-770, 1974.

Larichev, V. D. and Reznik, G. M.: Two-dimensional solitary Rossby waves, Rep. Doklady USSR. Akad. Sci., 231, 10771080, 1976.

Larichev, V. D. and Reznik, G. M.: Numerical experiments on the study of collision of two-dimensional solitary Rossby waves, Rep. Doklady USSR. Akad. Sci., 264, 229-233, 1982. 
Larichev, V. D. and Reznik, G. M.: Colliding two-dimensional solitary Rossby waves, Oceanologia., 23, 725-734, 1983.

Makino, M., Kamimura, T., and Taniuti, T.: Dynamics of twodimensional solitary vortices in low $\beta$-plasma with convective motion, J. Phys. Soc. Japan, 50, 980-989, 1981.

McWilliams, J. C.: An application of equivalent modons to atmospheric blocking, Dyn. Atmos. Oceans, 5, 43-46, 1980.

McWilliams, J. C., Flierl, G. R., Larichev, V. D., and Reznik, G. M.: Numerical studies of barotropic modons, Dyn. Atmos. Oceans, 5 , 219-238, 1981.

McWilliams, J. C. and Zabusky, N. J.: Interactions of isolated vortices. I: Modons colliding with modons, Fluid Dyn., 19, 207 227, 1982

Mezinger, F. and Arakawa, A.: Numerical models used in atmospheric models, GARP Publication series, 17, 1976.

Nguyen Duc, J.-M. and Sommeria, J.: Experimental characterization of steady two-dimensional vortex couples, J. Fluid Mech., 192, 175-192, 1988.

Nycander, J.: Refutation of stability proofs for dipole vortices, Phys. Fluids, A4, 467-476, 1992.

Orlandi, P., Verzicco, R., and van Heijst, G. J. F.: Stability of shielded vortex dipoles, In: Modelling of Oceanic Vortices, (Ed) van Heijst, G. J. F., North Holland, Amsterdam, 169-176, 1994.

Pakyari, A. and Nycander, J.: Steady two-layer vortices on the betaplane, Dyn. Atmos. Oceans, 25, 67-86, 1996.

Reznik, G. M.: An exact solution for the two-dimensional topographic solitary wave. Doklady Akad. Nauk SSSR, 285, 981-
985, 1985.

Reznik, G. M. and Sutyrin, G. G.: Baroclinic topographic modons, J. Fluid Mech., 437, 121-142, 2001.

Samarsky, A. A.: Theory of finite-difference schemes, Moskow, Nauka, 616 pp., 1989.

Stern, M. E.: Minimal properties of planetary eddies, J. Mar. Res., $33,1-13,1975$

Tribia, J. J.: Modons in spherical geometry, Geophys. Astrophys. Fluid Dynam., 30, 131-168, 1984.

van Heijst, G. J. F. and Flór, J. B.: Dipole formation and collisions in a stratified fluid, Nature, 340, 212-215, 1989.

van Geffen, J. H. G. M and van Heijst, G. J. F.: Viscous evolution of 2D dipolar vortices, Fluid Dyn. Res., 22, 191-213, 1998.

Verkley, W. T. M.: The construction of barotropic modons on a sphere, J. Atmos. Sci., 41, 2492-2504, 1984.

Verkley, W. T. M.: Stationary barotropic modons in westerly background flows, J. Atmos. Sci., 44, 2383-2398, 1987.

Verkley, W. T. M.: Modons with uniform absolute vorticity, J. Atmos. Sci., 47, 727-745, 1990.

Verkley, W. T. M.: A numerical method to find form-preserving free solutions of the barotropic vorticity equation on a sphere, J. Atmos. Sci., 50, 1488-1503, 1993.

Velasco Fuentes, O. U. and van Heijst, G. J. F.: Experimental study of dipolar vortices on a topographic $\beta$-plane, J. Fluid Mech., 259, 79-106, 1994.

Zabusky, N. J. and McWilliams, J. C.: A modulated point vortex model for geophysical $\beta$-plane, Phys. Fluids, 2175-2182, 1982. 